\title{
Molecular Dynamics Simulation Study on the Mechanism of the Inhibition of ATP Hydrolysis with Inhibitors in Human Papillomavirus Type 18 E1 Helicase*
}

\author{
Xiuli Lu ${ }^{1,2}$, Yong Zhang ${ }^{1}$, Shuchao Chen ${ }^{1}$, Yang $\mathrm{Li}^{1}$, Dan Jia ${ }^{1}$, Weiqi Wang ${ }^{1}$, Bing Gao ${ }^{3}$ and Hongsheng Liu ${ }^{1,2}$ \\ ${ }^{1}$ School of Life Science, Liaoning University, Shenyang 110036, China \\ ${ }^{2}$ Research Center for Computer Simulating and Information Processing of Bio-macromolecules of Liaoning Province, Shenyang \\ 110036, China \\ ${ }^{3}$ Institute of Basic Medical Sciences, Shenyang Medical College, Shenyang, 110034, China \\ gaobing_cn@yahoo.com.cn
}

\begin{abstract}
The biphenylsulfonacetic acid and its derivatives were found to inhibit ATP hydrolysis by an allosteric mechanism involving tyrosine 486 of HPV6 E1 Helicase as well as tyrosine 492 of HPV 18 E1. A theoretical study on the binding conformations and allosteric function of these inhibitors has been carried out using docking analysis and molecular dynamics (MD) simulation. The appropriate binding orientations and conformations of the (biphenyl4-ylsulfonyl) acetic acid interacting with HPV 18 E1 were revealed by the docking study. The MD simulation results obtained from NAMD showed that the binding of (biphenyl-4-ylsulfonyl) acetic acid at the site of Tyr492 was stabilizing around the Lys490 of HPV18 E1, the active site of its ATP hydrolysis. And the protein structure near its predicted allosteric and active sites of HPV 18 E1 has been altered after the binding of the inhibitor to the protein E1 with the different second structure type and length, suggesting that this compound could change the structure conformation near the active center of E1, through which exerts its enzyme-inhibiting function. A series of biphenylsulfonacetic acid derivatives, the reported HPV18 E1 inhibitors, have been then analyzed by docking study. The results revealed that all these compounds could stably bind to the protein with a good binding free energy, suggesting these derivatives could exert a similar allosteric effect on the E1 protein. Taken together, these theoretical results can offer useful references for understanding the mechanisms of allosteric effect of these compounds and directing the molecular design of this kind of inhibitor with improved activity.
\end{abstract}

Index Terms - HPV E1, biphenylsulfonacetic acid, allosteric effect, molecular dynamics.

\section{Introduction}

Human papillomavirus (HPV), belonging to the papillomavirus family, which infect the squamous and mucosal epithelia of many different mammals including humans. For example, HPV1 causes plantar warts, HPV6 and -11 cause anogenital warts, and infection with HPV16 (50\% to 60\%) and $-18(10 \%-12 \%)$ can lead to cervical cancer and oropharyngeal cancer [1]. Of the HPV types which infect the anogenital region and are associated with cancer are collectively referred to as "high-risk" types, while those that cause only benign warts are known as "low-risk" types [2]. In spite of the medical progress associated with treating and screening for
HPV infections, an HPV-specific antiviral drug is still lacking, and there are only a few reports of HPV-specific inhibitors which could serve as potential leading compounds for drug discovery.

The small circular double-stranded DNA genome of papillomaviruses encodes only eight well-characterized proteins [3]. The E1 helicase is the most highly conserved protein and the only one with enzymatic activity [4,5]. As E1 is essential for viral replication and pathogenesis, it is an attractive target for the development of anti-HPV drugs [6,7]. Up to now, only a few E1 ATPase inhibitors have been reported. For example, it was recently reported on the discovery that a biphenylsulfonacetic acid inhibitor of HPV6 E1, by high-throughput screening of compound collection, which inhibits the ATPase activity of HPV6 E1 [8]. A series of E2 inhibitors targeting HPV DNA replication proteins have also been published [9]. These compounds have become the only potent and selective small molecules inhibiting HPV replications. E1 is the replicative helicase of HPV, which binds to the origin of replication in conjunction with the E2 protein [10]. The formation of the E1-E2-origin complex involves not only the binding of both proteins to specific DNA elements in the origin but also a protein-protein interaction between the N-terminal transactivation domain of E2 and the helicase/ATPase domain of E1 [11]. Assembly of the E1-E2ori complex facilitates the recruitment of additional E1 molecules to the origin, which assemble into hexamers in a reaction that is stimulated by ATP binding [12]. The results demonstrated that ATP is the power for the helicase activity of E1 and acts as a molecular trigger during the initiation of viral DNA replication to help sever the interaction of E1 with E2 and promote its hexamerization at the origin [13]. It was reported that the biphenylsulfonacetic acid and its derivatives inhibit ATP hydrolysis by an allosteric mechanism involving tyrosine 486 of HPV6 E1 helicase as well as HPV 18 E1, which probably because of the tyrosine 486 of HPV6 conserved at tyrosine 492 of HPV 18 E1 [14]. How do biphenylsulfonacetic acid inhibitors inhibit ATP hydrolysis of E1 by the allosteric effect, however, has not been well studied.

\footnotetext{
* This work was supported by the grant of the construction of public service platform of Shenyang city (No.F10-188-8-00).
} 
We speculate that the biphenylsulfonacetic acid inhibitors must bind to a pocket near tyrosine 486 of E1 and thus impair the ATP binding allosterically. The crystal structure of the HPV18 E1 helicase domain has recently been reported [15], and it is interesting to use this structure to predict the location of the biphenylsulfonacetic acid binding site on the E1 protein. In the present study, we explored the kinetic mechanism of the binding actions of these compounds to E1, utilizing the molecular dynamics simulation analysis.

\section{Methods}

\section{A. Docking study}

The AutoDock4.2 program [16,17] was used for exploring the interaction between (biphenyl-4-ylsulfonyl) acetic acid and E1 by molecular docking. Crystalline structure of HPV18 E1 (PDBID:1TUE) was downloaded from Protein Data Bank and prepared for docking [18]. The Lamarckian Genetic Algorithm was used for docking experiments [16]. Binding sites were set around an area in which Tyr 492 and Lys 490 was contained. Dimensions of $64 \times 50 \times 44$ points with the spacing of $0.375 \AA$ was applied while grid centre coordinates were set to 28.807 , $32.339,125.869$ respectively. During the docking process, the ligand was set to be rotatable and the receptor was fixed. 100 docking runs were performed with the initial population of 150 individuals for the complex. Maximum number of generations and energy evaluation were set to 27,000 and $250,000,000$ respectively. Binding energy was evaluated by semi-empirical free energy evaluation function. Then the complex consisting of (biphenyl-4-ylsulfonyl) acetic acid and E1 was saved with MGLTools for further study of molecular dynamics simulation.

\section{B. Molecular dynamics}

The molecular dynamics (MD) simulation was performed with the software NAMD [19]. The electrostatic interactions were processed with the Particle Mesh Ewald (PME) method. The crystal structure of E1-E2 was taken for MD simulation calculations in the isobaric, isothermal ensemble $(310 \mathrm{~K})$, an integration time step of $2 \mathrm{fs}$ and the coordinates of system were stored every 1 ps. $10 \mathrm{~ns}$ MD simulation was performed to explore the interaction between receptor and ligand. All the analyses of the trajectory were performed by VMD 1.9.1 program [20].

\section{Selection of representative snapshots}

Although all the frames were saved during the MD simulation, a portion of frames were extracted to represent the whole conformational change of the protein during simulation. With VMD 1.9.1, the root mean-square deviation (RMSD) of backbone of complex protein was calculated, so we could know whether it was stable or not at the end of 10ns, we also made a comparison between the complex and macromolecule alone.

\section{Results}

A. Analysis of docking between HPV18 E1 and inhibitors

The Tyr-486 in HPV6 E1 identified previously (corresponding to Tyr-492 in HPV18) is close to the ATP binding site which has highly conserved Lys-484 (Lys-490 in HPV18) and essential for catalysis of ATP [21]. The crystal structure of the HPV18 E1-E2 can be used for exploring the location of the biphenylsulfonacetic acid binding site on E1 protein. Guided by structural information, it may be possible to explain the corresponding mechanism in the type of HPV18, because the data showed that the tyrosine and lysine are on the same $\alpha$-helix. Inhibitors interacting with the tyrosine and the other residues nearby could probably cause a conformational change that would affect the binding of ATP, which might induce the inhibition of E1 activity. To confirm this hypothesis at the atomic level, we performed the molecular docking and analysis to identify the possible interaction of inhibitors and the E1 protein. We set box center at Tyr-492 in HPV18 E1 protein in docking study, which makes it possible to explore the effect of the biphenylsulfonacetic acid inhibitors on the conformational change of E1 at the atomic level. By ADT [22], we probed into the quality of the binding between E1 and (biphenyl-4-ylsulfonyl) acetic acid from binding energy and cluster analysis (Fig. 1). From the ADT, the binding energy of complex is $-5.6 \mathrm{kcal} / \mathrm{mol}$, which means there is an interaction between the inhibitor and E1 protein, and the inhibitor could be stable around Tyr492 so that might change the conformation of E1 and affect the binding of ATP.

\section{B. Molecular dynamics simulation}

Considering the key importance of (biphenyl-4-ylsulfonyl) acetic acid in the activity domain, the structure of E1-E2 was taken as an initial structure for MD simulation studies. Two simulations of HPV18 E1-E2 either with or without (biphenyl4-ylsulfonyl) acetic acid were performed for $10 \mathrm{~ns}$ until the system was stabilized with no significant changes after $8 \mathrm{~ns}$. The stability of system was evaluated based on the analyses of the root mean square deviation (RMSD) of backbone (Fig. 2a) and the total energy of the protein (Fig. 2b and c). Both analyses revealed that the systems were relatively stabilized thus affirming that the simulation is reliable with no artifacts. It also indicated that the binding of (biphenyl-4-ylsulfonyl) acetic acid to the site of Tyr492 was stabilizing around the Lys490, the enzyme-active site of E1.

Based on the RMSD and total energy, we get the stable structure of complexes either in presence or absence of (biphenyl-4-ylsulfonyl) acetic acid by VMD 1.9.1 after the molecular dynamics simulations performed for 10ns. From the snapshots, we found that the conformation of protein altered after the binding of the (biphenyl -4-ylsulfonyl) acetic acid to the protein, if compared to that before. We found some $\alpha$-helix and $\beta$-sheets located around Tyr492 and Lys 490 by aligning two conformations with VMD 1.9.1(Fig. 3). The analysis of sequences revealed that the $\alpha$-helix and $\beta$-sheets around Lys490 contained different residues respectively. As the result of the conformational change, the protein could be different between the two groups in terms of both second structure type and length (shown in Table I). 
(a)

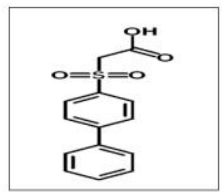

(b)

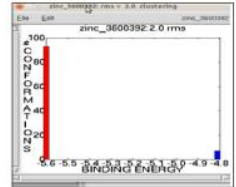

(c)

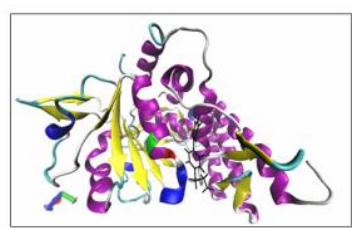

Fig. 1 The docking results of E1-E2 and (biphenyl-4-ylsulfonyl) acetic acid. The molecular formula of (biphenyl-4-ylsulfonyl) acetic acid (a). The binding energy in docking study (b).Docking conformation is shown in picture (c).

(a)

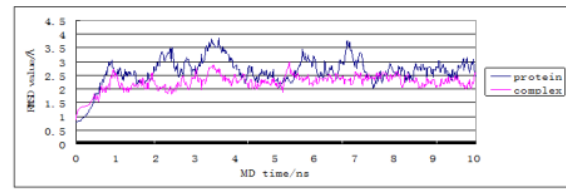

(b)

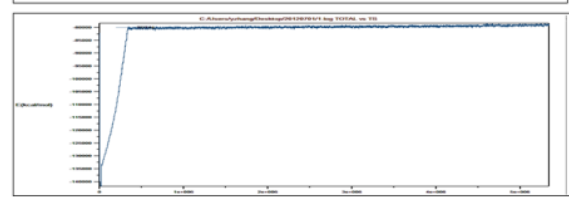

(c)

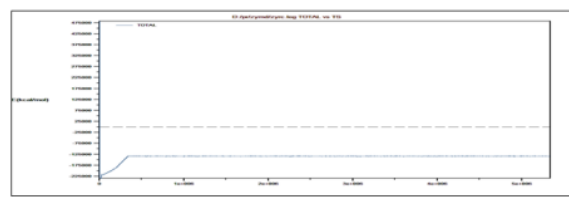

Fig. 2 RMSD for HPV18 E1-E2 either in absence or presence of (biphenyl4-ylsulfonyl) acetic acid (a) and two sets of conformational total energy graphs $(\mathrm{b}, \mathrm{c})$ for the $10 \mathrm{~ns}$ MD simulation.
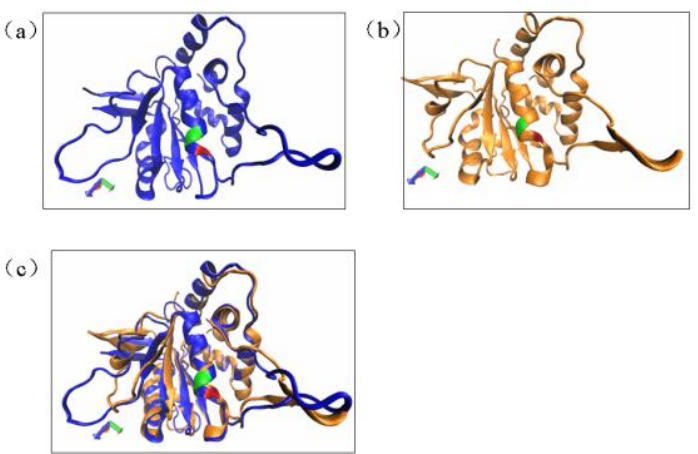

Fig. 3 The conformations of protein (a) and complex (b) in stable environment at the same time (green mark is Tyr492, red mark is Lys490, a and b). The alignment for both conformations(c).

Based on the above, docking recognition play a key role in the binding of (biphenyl-4-ylsulfonyl) acetic acid on the Tyr492 of protein. It can be speculated that the compound induced conformational alteration of protein from the MD simulation results. The change of second structure can be a critical factor to affect the ATP binding on the site of Lys490. Overall, our MD simulation studies of E1-E2 protein either in the presence or absence of (biphenyl-4-ylsulfonyl) acetic acid has proved the allosteric mechanism of ATPase inhibitor during conformational change.

C. The docking of Biphenylsulfonacetic Acid derivatives to the HPV18 E1

Based on the report which revealed biphenylsulfonacetic acid inhibitors can inhibit ATP hydrolysis in human papillomavirus type $6 \mathrm{E} 1$ helicase, we know that the series of Biphenylsulfonacetic Acid derivatives might inhibit ATP hydrolysis. We finally conducted the docking study of them as well as (biphenyl-4-ylsulfonyl) acetic acid (shown in Table II). By analyzing the results of docking, we found the series of biphenylsulfonacetic acid derivatives could bind with the protein fairly well with a good binding free energy, suggesting that the Lys492 residue of HPV18 E1 might be a new screening active site for the in silico virtual screening of the HPV replication inhibitors.

TABLE I The comparison between protein and complex in second structure type and length

\begin{tabular}{|l|l|l|l|l|}
\hline & Protein & & complex & \\
\hline Second structure & & Length & & Length \\
\hline Turn & $\begin{array}{l}\text { Thr474- Lys477 } \\
\text { Pro485- Thr488 }\end{array}$ & $\begin{array}{l}29.22 \\
33.75\end{array}$ & Thr474- Asn478 & 30.05 \\
\hline $\begin{array}{l}\text { Extend } \\
\text { conformation }\end{array}$ & Cys479- Cys483 & 160.20 & Cys479- Cys483 & 179.94 \\
\hline Alpha helix & Lys490- Ile501 & 288.67 & Lys490- Ile501 & 279.02 \\
\hline 3-10 helix & Ala504- Ile506 & 41.30 & $\begin{array}{l}\text { Ala486- Thr488 } \\
\text { Gly503- Val505 }\end{array}$ & 29.76 \\
\hline Coil & $\begin{array}{l}\text { Asn478 } \\
\text { Gly484 } \\
\text { Gly489 } \\
\text { Gln502- Gly503 }\end{array}$ & 14.53 & $\begin{array}{l}\text { Gly484- Pro485 } \\
\text { Gln502 }\end{array}$ & 14.83 \\
\hline
\end{tabular}

TABLE II The potential of inhibitor against HPV18 E1-E2

\begin{tabular}{|c|c|c|c|}
\hline \multirow[t]{2}{*}{ Name } & \multirow{2}{*}{$\begin{array}{l}\text { Binding energy } \\
(\mathrm{kcal} / \mathrm{mol})\end{array}$} & \multicolumn{2}{|l|}{ IC50 } \\
\hline & & HPV6a & HPV18 \\
\hline (biphenyl-4-ylsulfonyl) acetic acid & -5.60 & $2000 \pm 400$ & $3600 \pm 1300$ \\
\hline $\begin{array}{l}\text { ( }\left\{4^{\prime}-[2-\text { oxo-2- }\right. \\
\text { (phenylamino)ethyl]biphenyl-4- } \\
\text { yl }\} \text { sulfonyl)acetic acid }\end{array}$ & -7.68 & $160 \pm 40$ & $620 \pm 70$ \\
\hline $\begin{array}{l}\left(\left\{3^{\prime}-[(2-\right.\right. \\
\text { methylbenzyl)carbamoyl]biphenyl- } \\
\text { 4-yl }\} \text { sulfonyl }) \text { acetic acid }\end{array}$ & -7.13 & $32 \pm 4$ & $11000 \pm 20$ \\
\hline $\begin{array}{l}\text { (\{3'-[(naphthalen-1- } \\
\text { ylmethyl)carbamoyl]biphenyl-4- } \\
\text { yl }\} \text { sulfonyl)acetic acid }\end{array}$ & -7.13 & $4.3 \pm 1.1$ & $2300 \pm 40$ \\
\hline
\end{tabular}




\section{Discussion}

In the present, we studied the allosteric mechanism of the inhibition of ATP hydrolysis of HPV18 replication inhibitors. This methodology including analysis of the dynamic motion of the protein, then developing protein-based structure to retrieve hits and finally performing docking into plausible conformations would be a reliable procedure to apply in identifying ATP hydrolysis.

The residue tyrosine 486 in HPV6 E1 (corresponding to Tyr-492 in HPV18), is close to the ATP binding site which locates in the same helix as the highly conserved Lys-484 (Lys-490 in HPV18), interacts with ATP and is essential for catalysis [21]. Consistent with the allosteric mechanism observed in previous study, inhibitors interacting with this crucial tyrosine could also cause a conformational change that would affect the binding of ATP. Guided by structural information, it may now be possible to develop highly active compounds which might suppress HPV6 and -11 E1 as well as HPV18, even the other high-risk related proteins. Although these novel ATPase inhibitors presented in our work currently lack the necessary validation of physicochemical properties both in vitro and in vivo, validation to be good drug candidates, they apparently show the possibly greater potential of compounds in silico which could used as better drug candidates for targeting E1 ATPase and helicase, which thus could be designed as more effective antivirals for HPV.

\section{Acknowledgment}

We hold great gratitude to our colleagues from Technology Center for Computer Simulation of Biological Macromolecule and Information Processing Engineering of Liaoning Province for helping us with respect to simulation technology.

\section{References}

[1] Gillison ML, "Human papillomavirus-associated head and neck cancer is a distinct epidemiologic, clinical, and molecular entity," Semin Oncol, vol. 31, pp. 744-754, 2004.

[2] zur HH, de Villiers EM, "Human papillomaviruses," Annu Rev Microbiol, vol. 48, pp. 427-447, 1994.

[3] Longworth MS, Laimins LA, "Pathogenesis of human papillomaviruses in differentiating epithelia," Microbiol Mol Biol Rev, vol. 68, pp. 362372, 2004.

[4] Stenlund A, "Initiation of DNA replication: lessons from viral initiator proteins," Nat Rev Mol Cell Biol, vol. 4, pp. 777-785, 2003.

[5] Wilson VG, West M, Woytek K, Rangasamy D, "Papillomavirus E1 proteins: form, function, and features," Virus Genes, vol. 24, pp. 275$290,2002$.
[6] Phelps WC, Barnes JA, Lobe DC, "Molecular targets for human papillomaviruses: prospects for antiviral therapy," Antivir Chem Chemother, vol. 9, pp. 359-377, 1998.

[7] Sterlinko GH, Banks L, "HPV proteins as targets for therapeutic intervention," Antivir Ther, vol. 9, pp. 665-678, 2004.

[8] White PW, Faucher AM, Massariol MJ, Welchner E, Rancourt J, Cartier M, Archambault J, "Biphenylsulfonacetic acid inhibitors of the human papillomavirus type 6 E1 helicase inhibit ATP hydrolysis by an allosteric mechanism involving tyrosine 486," Antimicrob Agents Chemother, vol. 49, pp. 4834-4842, 2005.

[9] White PW, Titolo S, Brault K, Thauvette L, Pelletier A, Welchner E, Bourgon L, Doyon L, Ogilvie WW, Yoakim C, Cordingley MG, Archambault J, "Inhibition of human papillomavirus DNA replication by small molecule antagonists of the E1-E2 protein interaction," J Biol Chem, vol. 278, pp. 26765-26772, 2003.

[10] Frattini MG, Laimins LA, "Binding of the human papillomavirus E1 origin-recognition protein is regulated through complex formation with the E2 enhancer-binding protein," Proc Natl Acad Sci U S A, vol. 91, pp. 12398-12402, 1994.

[11] Abbate EA, Berger JM, Botchan MR, "The X-ray structure of the papillomavirus helicase in complex with its molecular matchmaker E2," Genes Dev, vol. 18, pp. 1981-1996, 2004.

[12] Fouts ET, Yu X, Egelman EH, Botchan MR, "Biochemical and electron microscopic image analysis of the hexameric E1 helicase," J Biol Chem, vol. 274, pp. 4447-4458, 1999.

[13] Sanders CM, Stenlund A, "Recruitment and loading of the E1 initiator protein: an ATP-dependent process catalysed by a transcription factor," EMBO J, vol. 17, pp. 7044-7055, 1998.

[14] Wang Y, Coulombe R, Cameron DR, Thauvette L, Massariol MJ, Amon LM, Fink D, Titolo S, Welchner E, Yoakim C, Archambault J, White PW, "Crystal structure of the E2 transactivation domain of human papillomavirus type 11 bound to a protein interaction inhibitor," J Biol Chem, vol. 279, pp. 6976-6985, 2004.

[15] Titolo S, Brault K, Majewski J, White PW, Archambault J, "Characterization of the minimal DNA binding domain of the human papillomavirus e1 helicase: fluorescence anisotropy studies and characterization of a dimerization-defective mutant protein," J Virol, vol. 77, pp. 5178-5191, 2003.

[16] Garrett MM, David SG, Robert SH, Ruth H, WEH, Richard KB, Arthur JO, "Automated docking using a Lamarckian genetic algorithm and an empirical binding free en ergy function," J Comput Chem, 1998.

[17] Park H, Lee J, Lee S, "Critical assessment of the automated AutoDock as a new docking tool for virtual screening," Proteins, vol. 65, pp. 549554, 2006.

[18] Abbate EA, Berger JM, Botchan MR, "The X-ray structure of the papillomavirus helicase in complex with its molecular matchmaker E2," Genes Dev, vol. 18, pp. 1981-1996, 2004.

[19] Phillips JC, Braun R, Wang W, Gumbart J, Tajkhorshid E, Villa E, Chipot C, Skeel RD, Kale L, Schulten K, "Scalable molecular dynamics with NAMD," J Comput Chem, vol. 26, pp. 1781-1802, 2005.

[20] Humphrey W, Dalke A, Schulten K, "VMD: visual molecular dynamics," J Mol Graph, vol. 14, pp. 33-38, 1996.

[21] Gorbalenya AE, E.V.Koonin, "Helicases: amino acid sequence comparisons and structure-function relationships," Curr Opin Struct Biol, 1993.

[22] Sanner MF, "Python: a programming language for software integration and development," J Mol Graph Model, vol. 17, pp. 57-61, 1999. 